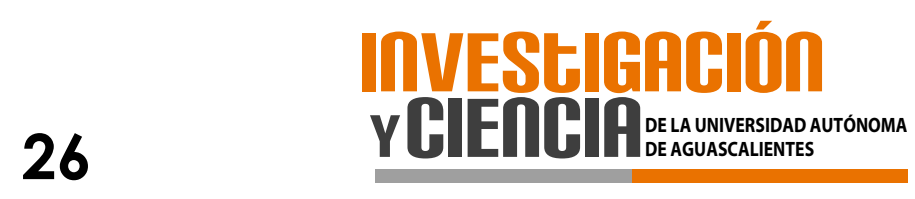

\title{
Comportamiento poscosecha en girasol (Helianthus annuus L.) en función de soluciones pulso
}

\author{
Postharvest behavior in sunflower (Helianthus annuus L.) \\ as a function of pulse solutions
}

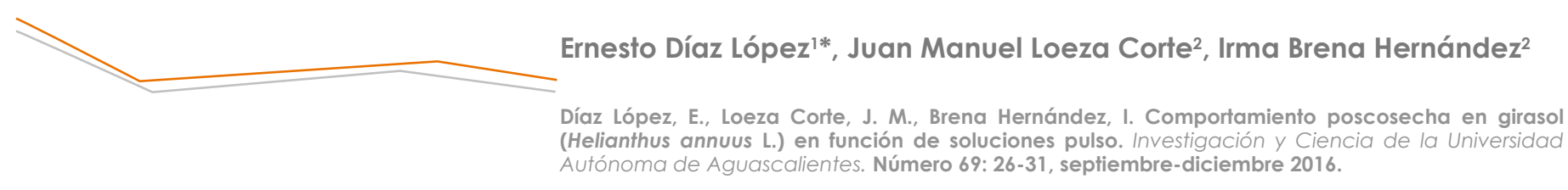

RESUMEN to determine the influence of the sucrose solution, citric acid, hydroxyquinoline and giberelic acid on the vase life of sunflower. The experiment was carried

Los cultivos florícolas son importantes, ya que generan divisas y mano de obra; en especies como el girasol el conocimiento sobre su manejo en poscosecha es limitado. El objetivo del presente estudio fue determinar la influencia de la solución de sacarosa, ácido cítrico, hidroxiquinoleína y ácido giberélico sobre la vida de florero de girasol. El experimento se llevó a cabo bajo condiciones de laboratorio en Teotitlán de Flores Magón, Oaxaca, México, durante el verano de 2014. Los resultados indican que la solución compuesta con ácido cítrico, sacarosa, hidroxiquinoleína y pH de 5.5 a 5.7 obtuvo la mayor vida de florero con lígulas turgentes, mientras que la aplicación de ácido giberélico a $\left(50 \mathrm{mg} \mathrm{l}^{-1}\right)$ impidió la apertura de la inflorescencia.

\section{ABSTRACT}

The floriculture crops are important because they generate foreign exchange and labor; in the species such as sunflower knowledge of postharvest handling is limited. So, the objective of this study was

Palabras clave: ácido giberélico, ácido cítrico, hidroxiquinoleína, sacarosa, poscosecha.

Keywords: giberelic acid, citric acid, hidroxyquinoline, sacarose, posharvest.

\footnotetext{
Recibido: 8 de abril de 2015, aceptado: 13 de mayo de 2016

Ingeniería en Agricultura Sustentable y Protegida, Universidad Tecnológica de Tehuacán.

2 Ingeniería en Agroindustrias, Universidad de la Cañada.

* Autor para correspondencia: lernesto@colpos.mx
} under laboratory conditions in Teotitlan Flores Magon, Oaxaca, Mexico, during the summer of 2014. The results indicate that the compound with citric acid solution, saccharose, hydroxyquinoline and $\mathrm{pH}$ of 5.5 at 5.7 obtained the largest vase life with turgid ligules, while the application of gibberellic acid $\left(50 \mathrm{mg} \mathrm{l}^{-1}\right)$ prevented the opening of the inflorescence.

\section{INTRODUCCIÓN}

México es un país que dispone de una diversidad en climas y microclimas favorables para la producción florícola. Especies como el girasol (Helianthus annuus L.) presentan gran potencial para ser explotadas como ornamentales, aunque esta especie es más conocida como oleaginosa por la producción de aceite de sus semillas (Morales et al., 2007). En la actualidad ha cobrado importancia por la belleza de su capítulo, lo que ha conducido a su utilización en arreglos florales, pero los estudios al respecto son limitados, por lo que se requieren aportes en este rubro. El manejo en poscosecha de cultivos ornamentales tiene como principal objetivo prolongar el tiempo después del corte, lo que se ha denominado vida de florero (Juárez et al., 2011; Leyva et al., 2011) y existen varias técnicas para lograr dicho objetivo, que consisten en el manejo de fertilizaciones a base de $\mathrm{N},\left(\mathrm{P}_{2} \mathrm{O}_{5}\right),\left(\mathrm{K}_{2} \mathrm{O}\right)$, así como la aplicación de fertilizaciones foliares a base de micronutrimentos (Rubio et al., 2007). 
Otra forma es la elaboración de soluciones pulso basadas en la aplicación de sacarosa que incrementan la vida de florero y la apertura floral, como se ha hecho en Rosa cultivares Grand Gala y Vega (Flores Ruvalcaba et al., 2005; Hernández Hernández et al., 2009). Otro modo es mediante la aplicación de soluciones a base de ácido giberélico $\left(\mathrm{AG}_{3}\right)$ que además mejoran la apariencia del follaje en especies como Dracaena marginata (Cruz Crespo et al., 2006; Trujillo Villagarcía et al., 2006; Bhattacharya et al., 2010; Alia Tejacal et al., 2011), este fitorregulador no solo se ha aplicado en cultivos ornamentales, también es conocido el efecto que tiene sobre algunos frutales como el mango, donde su aplicación en dosis de $25 \mathrm{mg} \mathrm{t}^{-1}$ retrasa la floración en este frutal (Pérez Barraza et al., 2008). Además, el uso y la aplicación de bactericidas aplicados a soluciones pulso, como hidroxiquinoleína (8HQC) controlan de manera efectiva el desarrollo de microorganismos que taponan los sistemas de conducción como el xilema y el floema y dan como resultado un incremento en la vida de florero en diversas especies florales de corte (Sun et al., 2001; Salisbury y Ross, 2003; Rebolledo et al., 2007). Estas técnicas ayudan a minimizar los costos de poscosecha para los productores, pero desde esta perspectiva el manejo en poscosecha en girasol de alguna forma es limitado. El objetivo del presente estudio fue evaluar la aplicación de diferentes soluciones basadas en sacarosa, ácido cítrico, ácido giberélico $\left(\mathrm{AG}_{3}\right)$ e hidroxiquinoleína 8-HQC sobre la vida de florero del girasol ornamental (Helianthus annuUs L.) CV. Victoria.

MATERIALES Y MÉTODOS

El trabajo fue realizado en condiciones de laboratorio, con la temperatura máxima, mínima y humedad relativa diarias durante el experimento en el verano de 2014 en Teotitlán de Flores Magón, Oaxaca, México, ubicado a $18^{\circ} 06^{\prime} \mathrm{N}, 97^{\circ} 06^{\prime} \mathrm{W}$ y 880 m.s.n.m., con inflorescencias de girasol en la etapa fenológica $\mathrm{R}_{4^{\prime}}$ la cual se caracteriza porque las flores liguladas son visibles en el interior de las brácteas del capítulo (De Caram et al., 2007), estas fueron sembradas en bolsas de polietileno con capacidad de $2 \mathrm{~kg}$, en una proporción de 2:1 (v/v) con suelo de la zona y hojarasca. El suelo corresponde a un lluvisol con pH de 6.7, contenido de materia orgánica de $1.7 \%$ y conductividad eléctrica de $2.1 \mathrm{mS} \mathrm{cm}^{-1}$. El genotipo de girasol utilizado en el experimento fue cv. Victoria, proporcionado por el banco de germoplasma del área de ecofisiología de cultivos del Colegio de Postgraduados.

Las inflorescencias fueron cortadas en campo a las 8:00 a.m. para evitar la deshidratación y fueron colocadas por un periodo de $24 \mathrm{~h}$ en agua corriente limpia con ajuste de $\mathrm{pH}$ de 5.5-5.7 con ácido sulfúrico al $5 \mathrm{~N}$. El experimento consistió en evaluar concentraciones de sacarosa, ácido cítrico, hidroxiquinoleína y ácido giberélico, donde los tratamientos fueron: testigo $\left(T_{0}\right)$, que consistió en agua corriente ajustando el pH entre 5.5 y $5.7 ;\left(\mathrm{T}_{1}\right)$, solución de sacarosa a $0 \%$ con ácido cítrico a 100 $\mathrm{mg} \mathrm{t}^{-1}+$ hidroxiquinoleína a $10 \mathrm{mg}^{-1} ;\left(\mathrm{T}_{2}\right)$, solución de sacarosa a $1 \%$ con ácido cítrico a $100 \mathrm{mg} \mathrm{t}^{-1}+$ hidroxiquinoleína a $10 \mathrm{mg}^{-1} ;\left(T_{3}\right)$, solución de sacarosa a $2 \%$ con ácido cítrico a $100 \mathrm{mg} \mathrm{t}^{-1}+$ hidroxiquinoleína a $10 \mathrm{mg} \mathrm{t}^{-1} ;\left(\mathrm{T}_{4}\right)$, sacarosa a $2 \%$ con ácido cítrico a $100 \mathrm{mg} \mathrm{t}^{t^{-1}}+$ ácido giberélico a $50 \mathrm{mg}^{t^{-1}}$ (Tabla 1). En todos los tratamientos el $\mathrm{pH}$ fue ajustado a un rango de 5.5 a 5.7 .

El experimento fue analizado mediante un diseño completamente aleatorizado (DCA) con cuatro repeticiones $(5 \times 4)=20$, un total de 20 unidades experimentales; lo que se constituyó por un florero de plástico de 1 I de capacidad y una inflorescencia de girasol por florero. El tallo con la inflorescencia en todos los tratamientos tuvo una longitud de $45 \mathrm{~cm}$ y para evitar sesgo en el consumo de agua y transpiración se eliminaron los nomófilos. Las variables evaluadas fueron: agua transpirada (AT), calculada pesando cada unidad experimental cada $72 \mathrm{~h}$ por medio de la expresión $A T=P_{1}-P_{2}$ donde: $P_{1}$ y $P_{2}$ son los pesos de cada unidad experimental expresados en $\mathrm{g}$ en los tiempos 1 y 2 , respectivamente, con ayuda de una balanza analítica; vida de florero (VF), calculando los días que duraron las flores liguladas turgentes y la base del tallo sumergida en la solución en buenas condiciones; diámetro de capítulo (DC), midiendo con ayuda de un vernier Truper 14388 estándar milimétrico; dicho diámetro fue medido en forma de cruz para obtener el promedio correspondiente.

A las variables respuesta que resultaron significativas se les aplicó la prueba de comparación de promedios de Tukey a un nivel de significancia de $5 \%$. 
Tabla 1. Tratamientos y concentración de soluciones aplicadas al girasol ornamental (Helianthus annuus L.) cv. Victoria

\begin{tabular}{|c|c|c|c|c|}
\hline Tratamiento & Ac. cítrico & Sacarosa & Hidroxiquinoleína & Ac. giberélico \\
\hline & $\mathrm{mg} \mathrm{l}^{-1}$ & $\%$ & $\mathrm{mg} \mathrm{|}^{-1}$ & $\mathrm{mg} \mathrm{l}^{-1}$ \\
\hline $\mathrm{T}_{0}$ & 0 & 0 & 0 & 0 \\
\hline $\mathrm{T}_{1}$ & 100 & 0 & 10 & 0 \\
\hline $\mathrm{T}_{2}$ & 100 & 1 & 10 & 0 \\
\hline $\mathrm{T}_{3}$ & 100 & 2 & 10 & 0 \\
\hline $\mathrm{T}_{4}$ & 100 & 2 & 10 & 50 \\
\hline
\end{tabular}

$\mathrm{T}_{0^{\prime}}$ testigo (solo agua corriente); $\mathrm{T}_{1^{\prime}}$ ácido cítrico $100 \mathrm{mg} \mathrm{t}^{-1}+10 \mathrm{mg} \mathrm{t}^{-1}$ de hidroxiquinoleína; $T_{2^{\prime}}$ ácido cítrico $100 \mathrm{mg} \mathrm{t}^{-1}+1 \%$ de sacarosa $+10 \mathrm{mg} \mathrm{t}^{-1}$ de hidroxiquinoleína; $\mathrm{T}_{3}$, ácido cítrico $100 \mathrm{mg} \mathrm{l}^{-1}+$ sacarosa $2 \%$ y , ácido cítrico $100 \mathrm{mg} \mathrm{l}^{-1}+$ sacarosa $2 \%+50 \mathrm{mg} \mathrm{l}^{-1}$ de ácido giberélico. El pH se ajustó en todos los tratamientos en un rango de 5.5 a 5.7. Elaboración propia.

RESULTADOS

\section{Agua transpirada (AT)}

El agua transpirada por las inflorescencias de girasol fue mayor en los tratamientos $\mathrm{T}_{2}, \mathrm{~T}_{3}$ y $\mathrm{T}_{4}$ (Tabla 2), con una transpiración entre 35.2 y $40.3 \mathrm{~g}$ de agua; superaron al tratamiento $T_{1}$, el cual resultó igual que el testigo con 32.7 y $28.9 \mathrm{~g}$, respectivamente. Esta respuesta probablemente se debió a que los tratamientos $T_{2}, T_{3}$ y $T_{4}$ contenían concentraciones de sacarosa entre 1 y $2 \%$, lo que incrementó la actividad metabólica de las inflorescencias; es decir, la respiración por el consumo de carbohidratos, lo que produjo una mayor absorción y transpiración de agua (Figueroa et al., 2005).

Tabla 2. Agua transpirada en inflorescencias de girasol ornamental (Helianthus annuus L.) cv. Victoria

\begin{tabular}{|c|c|}
\hline Tratamiento & Agua transpirada (g) \\
\hline $\mathrm{T}_{0}$ & $28.9 \mathrm{~b}^{\mathbf{z}}$ \\
\hline $\mathrm{T}_{1}$ & $32.7 \mathrm{~b}$ \\
\hline $\mathrm{T}_{2}$ & $39.0 \mathrm{a}$ \\
\hline $\mathrm{T}_{3}$ & $35.2 \mathrm{a}$ \\
\hline $\mathrm{T}_{4}$ & $40.3 \mathrm{a}$ \\
\hline $\mathrm{DSH}$ & 6.28 \\
\hline $\mathrm{CV} \%$ & 2.96 \\
\hline
\end{tabular}

$\mathrm{T}_{0}$, testigo (solo agua corriente con $\mathrm{pH}$ ajustado 5.5 a 5.7); $\mathrm{T}_{1}$, ácido cítrico $100 \mathrm{mg} \mathrm{l}^{-1}+10 \mathrm{mg} \mathrm{t}^{-1}$ de hidroxiquinoleína; $\mathrm{T}_{2}$, ácido cítrico $100 \mathrm{mg} \mathrm{t}^{-1}+1 \%$ de sacarosa $+10 \mathrm{mg} \mathrm{l}^{-1}$ de hidroxiquinoleína; $\mathrm{T}_{3}$, ácido cítrico $100 \mathrm{mg} \mathrm{t}^{-1}$ + sacarosa $2 \%$ y $\mathrm{T}_{4}$, ácido cítrico $100 \mathrm{mg} \mathrm{t}^{-1}+$ sacarosa $2 \%+50 \mathrm{mg} \mathrm{t}^{-1}$ de ácido giberélico, tratamientos; DSH, diferencia significativa honesta; CV, coeficiente de variación; ztratamientos dentro de la columna con la misma letra, estadísticamente son iguales según Tukey $p \leq 0.05$. Elaboración propia.

\section{Vida de florero (VF)}

En la Tabla 3 se presentan los valores medios para vida de florero y se observa que existieron diferencias significativas entre los tratamientos, en los cuales $T_{1}$, $\mathrm{T}_{2}$ y $\mathrm{T}_{3}$ obtuvieron la mayor vida de florero: 12.00, 11.00 y 11.75 días, lo que superó a los tratamientos $\mathrm{T}_{4}$ y el testigo, que lograron una vida de 6.00 y 7.50 días, respectivamente. Así, los tratamientos $T_{1}, T_{2}$ y $\mathrm{T}_{3}$ aumentaron la vida de florero hasta 4 días en relación con el testigo; es decir, tuvieron $60 \%$ más vida de florero con respecto al testigo.

Tabla 3. Vida de florero en girasol ornamental (Helianthus annuUs L.) Cv. Victoria

\begin{tabular}{|c|c|}
\hline Tratamiento & Vida de florero (días) \\
\hline $\mathrm{T}_{0}$ & $7.50 \mathrm{~b}^{\mathbf{z}}$ \\
\hline $\mathrm{T}_{1}$ & $12.00 \mathrm{a}$ \\
\hline $\mathrm{T}_{2}$ & $11.00 \mathrm{a}$ \\
\hline $\mathrm{T}_{3}$ & $11.75 \mathrm{a}$ \\
\hline $\mathrm{T}_{4}$ & $6.00 \mathrm{~b}$ \\
\hline $\mathrm{DSH}$ & 1.30 \\
\hline $\mathrm{CV} \%$ & 12.00 \\
\hline
\end{tabular}

$\mathrm{T}_{0}$, testigo (solo agua corriente con pH ajustado 5.5 a 5.7 ); $\mathrm{T}_{1}$, ácido cítrico $100 \mathrm{mg} \mathrm{t}^{-1}+10 \mathrm{mg}^{-1}$ de hidroxiquinoleína; $\mathrm{T}_{2}$, ácido cítrico $100 \mathrm{mg} \mathrm{t}^{-1}+1 \%$ de sacarosa $+10 \mathrm{mg} \mathrm{t}^{-1}$ de hidroxiquinoleína; $\mathrm{T}_{3}$, ácido cítrico $100 \mathrm{mg} \mathrm{t}^{-1}$ + sacarosa $2 \%$ y $\mathrm{T}_{4}$, ácido cítrico $100 \mathrm{mg} \mathrm{t}^{-1}+$ sacarosa $2 \%+50 \mathrm{mg} \mathrm{H}^{-1} \mathrm{de}$ ácido giberélico; DSH, diferencia significativa honesta; CV, coeficiente de variación; "tratamientos dentro de la columna con la misma letra, estadísticamente son iguales según Tukey $p \leq 0.05$. Elaboración propia.

\section{Relación agua transpirada (AT) vs. vida de florero (VF)}

Con respecto a esta relación se puede apreciar que a medida que hay mayor transpiración se incrementa la vida de florero como ocurrió en los tratamientos $T_{1}, T_{2}$ y $T_{3}$; no así en el testigo y el tratamiento $T_{4^{\prime}}$ a pesar de presentar el mayor consumo de agua. 
Esta respuesta se debió a que la base del tallo floral se abrió y permitió mayor consumo de agua con una senescencia más rápida. En cambio, el testigo presentó menor transpiración porque el mismo no contó con la aplicación de sacarosa, lo que influyó en la fuente de energía limitada que las células tuvieron para lograr mayor vida de florero (Figura 1).

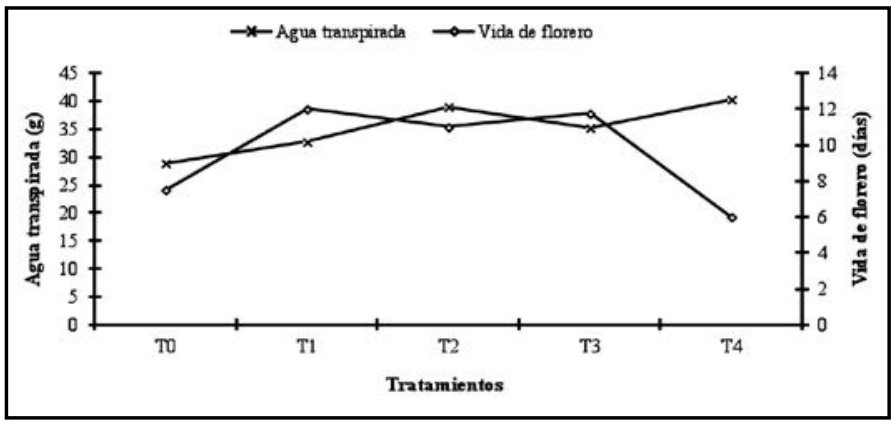

Figura 1. Relación entre vida de florero y agua transpirada en girasol (Helianthus annuus L.) cv. Victoria. Elaboración propia.

\section{Diámetro de capítulo (DC)}

En esta variable, el mayor diámetro de capítulo se observó en los tratamientos $T_{1}, T_{2}$ y $T_{3}$ con 9.20, 9.90 y $9.80 \mathrm{~cm}$, respectivamente, y superó al testigo, quien presentó un diámetro de $8.10 \mathrm{~cm}$. El menor diámetro ocurrió para $\mathrm{T}_{4^{\prime}}$ con $6.80 \mathrm{~cm}$ (Tabla 4), esto se atribuye al efecto del ácido giberélico $\mathrm{AG}_{3^{\prime}}$ cuya acción inhibe la iniciación floral en flores de corte y algunos frutales como el mango, a concentraciones que van en un rango de 50 a $250 \mathrm{mg} \mathrm{l}^{-1}$, lo que propició que el diámetro del capítulo de girasol tuviera un diámetro menor (Pérez Barraza et al., 2008).

Tabla 4. Diámetro de capítulo en girasol ornamental (Helianthus annuus L.) cv. Victoria

\begin{tabular}{|c|c|}
\hline Tratamiento & Diámetro $(\mathbf{c m})$ \\
\hline $\mathrm{T}_{0}$ & $8.10 \mathrm{c}^{\mathbf{z}}$ \\
\hline $\mathrm{T}_{1}$ & $9.20 \mathrm{a}$ \\
\hline $\mathrm{T}_{2}$ & $9.90 \mathrm{a}$ \\
\hline $\mathrm{T}_{3}$ & $9.80 \mathrm{a}$ \\
\hline $\mathrm{T}_{4}$ & $6.80 \mathrm{~d}$ \\
\hline $\mathrm{DSH}$ & 0.75 \\
\hline $\mathrm{CV} \%$ & 12.00 \\
\hline
\end{tabular}

$\mathrm{T}_{0}$, testigo (solo agua corriente con $\mathrm{pH}$ ajustado 5.5 a 5.7 ); $\mathrm{T}_{1}$, ácido cítrico $100 \mathrm{mg} \mathrm{l}^{-1}+10 \mathrm{mg} \mathrm{t}^{-1}$ de hidroxiquinoleína; $\mathrm{T}_{2}$, ácido cítrico $100 \mathrm{mg} \mathrm{t}^{-1}+1 \%$ de sacarosa $+10 \mathrm{mg} \mathrm{t}^{-1}$ de hidroxiquinoleína; $\mathrm{T}_{3}$, ácido cítrico $100 \mathrm{mg} \mathrm{t}^{-1}$ + sacarosa $2 \%$ y $\mathrm{T}_{4}$, ácido cítrico $100 \mathrm{mg} \mathrm{l}^{-1}+$ sacarosa $2 \%+50 \mathrm{mg} \mathrm{H}^{-1} \mathrm{de}$ ácido giberélico; DSH, diferencia significativa honesta; $\mathrm{CV}$, coeficiente de variación; ztratamientos dentro de la columna con la misma letra estadísticamente son iguales según Tukey $p \leq 0.05$. Elaboración propia.

\section{Efecto de la sacarosa (ES)}

La sacarosa provocó un efecto positivo para esta especie en concentraciones de 1 a $2 \%$, lo que resultó en el incremento de vida de florero, hasta por 4 días respecto al testigo y mayor diámetro de capítulo.

\section{Efecto del ácido giberélico $\left(\mathrm{AG}_{3}\right)$}

Al contrario de la sacarosa, el ácido giberélico tuvo un efecto negativo en el girasol, ya que el tratamiento donde se aplicó el mismo provocó que las flores tubulares del capítulo maduraran demasiado rápido en relación con las liguladas, además de que la base del tallo se destruyó en el florero (Figura 2).

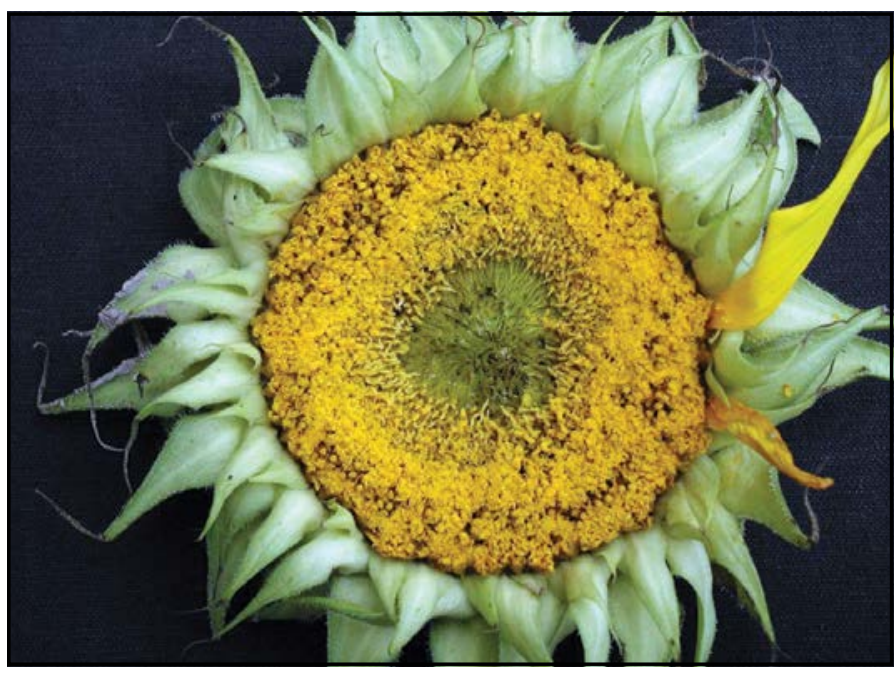

Figura 2. Efecto del ácido giberélico $\mathrm{AG}_{3}$ a $50 \mathrm{mg} \mathrm{l}^{-1}$ sobre girasol ornamental (Helianthus annuus L.) CV. Victoria. Fotografía tomada por los autores.

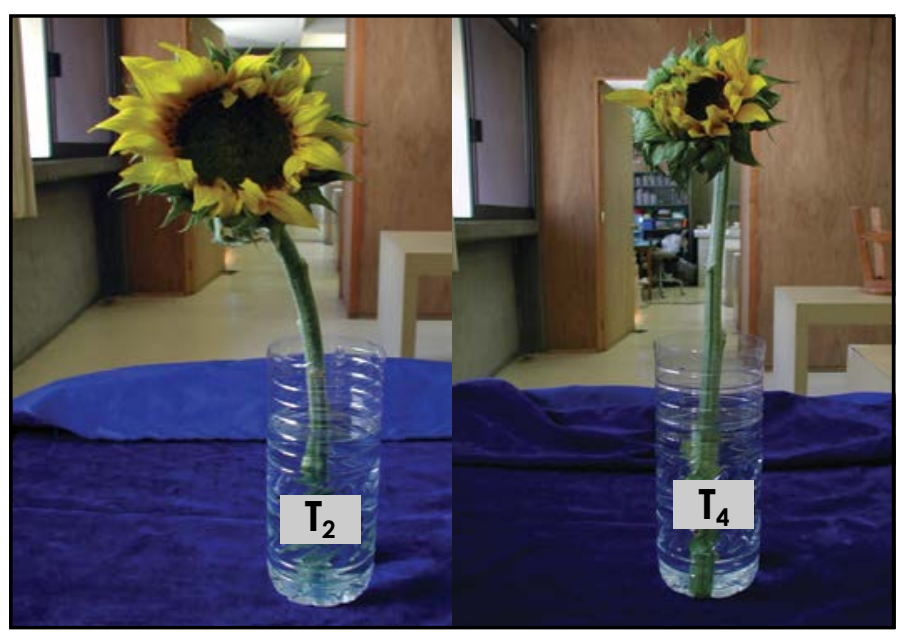

Figura 3. Efecto de la sacarosa a $1 \%\left(\mathrm{~T}_{2}\right)$ y efecto del ácido cítrico y $\mathrm{AG}_{3}$ en girasol (Helianthus annuus L.) cV. Victoria. Fotografías tomadas por los autores. 


\section{IIVESEIGGACIÓn

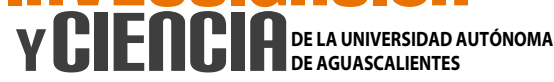

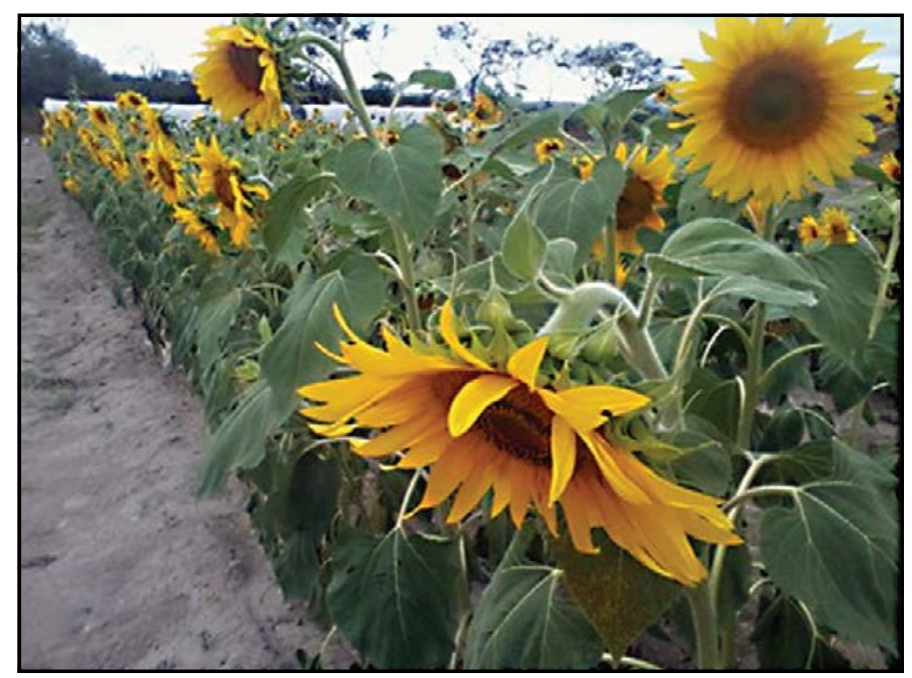

Figura 4. Girasol (Helianthus annuus L.) cv. Victoria en campo en la Universidad Tecnológica de Tehuacán, material que sirvió como germoplasma para evaluarse bajo condiciones de laboratorio. Fotografía tomada por Ernesto Díaz López.

\section{DISCUSIÓN}

Los tratamientos que contenían concentraciones de 1 y $2 \%$ de sacarosa presentaron una mayor agua transpirada quizá por mayor actividad metabólica de la inflorescencia, como indican Taiz y Zeiger (2015), quienes mencionan que la sacarosa a concentraciones en un rango de 1 a $4 \%$ incrementan la actividad respiratoria y transpiratoria de los vegetales, incluso en plantas de cultivo con alto nivel de fotosaturación, como el girasol.

En relación con la vida de florero, la mayor se presentó en $\mathrm{T}_{1}, \mathrm{~T}_{2}$ y $\mathrm{T}_{3}$; dicha respuesta se atribuye a la aplicación de 8-hidroxiquinoleína en la solución, molécula que actúa como biocida en los tratamientos, lo que ocasionó que disminuyera la proliferación de microorganismos que pudiesen tapar los conductos de xilema y floema; ello impidió un buen suministro de agua hacia las flores liguladas, lo que originó que las mismas se mantuvieran turgentes, como lo mencionan Hernández Fuentes et al. (2006).

De este modo, la relación agua transpirada vs. vida de florero está estrechamente relacionada, ya que los tratamientos que presentaron mayor cantidad de agua transpirada coincidieron con la mayor vida de florero, siempre y cuando la base del tallo estuviera en buenas condiciones, como se mencionó en el apartado de resultados. Estos hallazgos concuerdan con los reportados por Van Meeteren et al. (2006), quienes mencionan que la vida de florero en especies de la misma familia del girasol, como Crisantemo spp., se incrementa al agregar concentraciones de sacarosa de 1 a $2 \%$, las cuales funcionan como fuente de energía para procesos metabólicos como la respiración de las flores de corte en poscosecha, ya que estas no cuentan con un suministro de carbohidratos; por tal motivo, necesitan una fuente alterna de energía. También mencionan que al incrementar el agua transpirada por las flores liguladas del capítulo, estas presentan una buena apariencia, lo que le da a la inflorescencia un buen aspecto estético. Con respecto al ácido giberélico, los datos presentados en este estudio corresponden con los resultados reportados por Wei Ren et al. (2001), quienes al aplicar $\mathrm{AG}_{3}$ en Polianthus tuberosa no lograron la apertura de botones florales y concluyen que el ácido giberélico no induce la apertura floral en Polianthes.

CONCLUSIONES

Con base en los resultados obtenidos en la presente investigación se llegó a las siguientes conclusiones:

- Con una solución de pH 5.5 a 5.7 compuesta, con ácido cítrico (100 $\left.\mathrm{mg}^{-1}\right)$, sacarosa $(2 \%)$ e hidroxiquinoleína $10 \mathrm{mg} \mathrm{l}^{-1}$, se obtuvo la mayor vida de florero ( 11.75 días) en girasol cv. Victoria. La sacarosa a concentraciones de 1 y $2 \%$ tuvo efecto positivo en esta especie (Helianthus annuus L.), lo que se reflejó en un incremento de vida de florero de hasta 4.5 días con respecto al testigo.

- El ácido giberélico $\left(\mathrm{AG}_{3}\right)$ tuvo efecto negativo en el girasol a concentraciones de $50 \mathrm{mg} \mathrm{l}^{-1}$, lo que provocó que las flores tubulares maduraran rápidamente y dio como resultado que el capítulo no abriera completamente, lo que afectó el diámetro del mismo y la vida de florero.

- La hidroxiquinoleína incrementó la vida de florero en girasol al permitir mayor absorción de agua por el tallo hacia las flores tubulares y liguladas. 


\section{LITERATURA CITADA}

- ALIA TEJACAL, I. et al. Efecto de la aspersión de ácido giberélico en el crecimiento de cinco cultivares de nochebuena. Revista Mexicana de Ciencias Agrícolas, 3, 577-589, 2011.

- BHATTACHARYA, A. et al. Practical applications of manipulating plant architecture by regulating gibberellin metabolism. Journal of Plant Growth Regulation, 29(2): 249-256, 2010.

- CRUZ CRESPO, E. et al. Soluciones pulso en la calidad postcosecha de Lisianthus (Eustoma grandiflorum Raf.) cv. "Echo blue". Agricultura Técnica de México, 32(2): 191-200, 2006.

- DE CARAM, G. A. et al. Determinación de la curva de dilución de nitrógeno en diferentes fases fenológicas del girasol. Agricultura Técnica (Chile), 67(2): 189-195, 2007.

- $\quad$ FIGUEROA, l. et al. Cambios fisiológicos en postcosecha de dos cultivares de rosa con diferente duración en florero. Ciencia e Investigación Agraria, 32(3): 209-219, 2005.

- FLORES RUVALCABA, J. S. et al. Crecimiento vegetativo y floral del crisantemo [Dendranthema $x$ grandiflorum (Ramat) Kitamural] en respuesta a la presión osmótica de la solución nutritiva. Revista Chapingo serie Horticultura, 11 (2): 241-249, 2005.

- HERNÁNDEZ FUENTES, A. D. et al. Conservación de flores de alstroemeria (Alstroemeria spp.) mediante soluciones preservativas en postcosecha. Revista Chapingo Serie Horticultura, 12(1): 19-25, 2006.

- HERNÁNDEZ HERNÁNDEZ, F. et al. Diferencias anatómicas y uso de soluciones de pulso en dos cultivares de rosa (Rosa sp.). Revista Chapingo Serie Horticultura, 15(2): 11-16, 2009.

- JuÁREZ LÓPEZ, P. et al. Comportamiento fisiológico postcosecha de tallos florales de rosa (Rosa hybrida L.) en respuesta al fósforo aplicado en precosecha. Revista Bio Ciencias, 1 (2): 3-16, 2011.

- LEYVA OVALLE, O. R. et al. Polímero hidrofílico combinado con soluciones preservadoras en la vida de florero de tallos florales de rosa y heliconia. Tropical and Subtropical Agroecosystems, 13(3): 551-559, 2011.
- MORALES, R. E. et al. Producción de biomasa y rendimiento de semilla de girasol (Helianthus annuus L.)- frijol (Phaseolus vulgaris L.) en función del nitrógeno y fósforo. Ciencia Ergo sum, 14(2): 177-183, 2007.

- PÉREZ BARRAZA, M. H. et al. Uso de giberelinas para modificar crecimiento vegetativo y floración en mango "Tommy Atkins" y "Ataulfo". Revista Chapingo Serie Horticultura, 14 (2): 169-175, 2008.

- REBOlledo, C. et al. Almacenamiento de postcosecha y vida en florero de follaje juvenil de Eucalyptus gunnii Hook. F. (Myrtaceae) para uso en floristería. Agro Sur, 35(2): 32-34, 2007.

- RUBIO, G. et al. Distribución de nitrógeno, fósforo y azufre en un cultivo de colza: efectos sobre el ciclado de nutrientes. Ciencia del Suelo, 25(2): 189-194, 2007.

- SAlisBURY, F. B. y ROSS, C. W. Fisiología Vegetal (pp. 115-130). Grupo Editorial Iberoamérica, 2003.

- SUN, J. et al. Water relations and stamen abscission in cut flowers of selected myrtaceae. Acta Horticulturae, 543: 185$189,2001$.

- TAIZ, L. y ZEIGER, E. Plant physiology and development (pp. 432-435). US: Sinaver, 2015.

- tRUjillo VILLAGARCÍA, B. A. et al. Efecto del $\mathrm{CaCl}_{2}$ sobre la actividad enzimática antioxidante durante la vida de florero de gerbera (Gerbera Jamesonni H. Bolux Ex Hook F.). Revista Chapingo Serie Horticultura, 12(2): 203-209, 2006.

- VAN MEETEREN, U. et al. Inhibition of water uptake after harvesting cut flowers role of air emboli and wound-induced processes in chrysanthemum. Postharvest Biology and Tecnology, 41, 70-77, 2006.

- WEI REN, S. et al. Improvement of postharvest vase life and flower bud opening in Polianthes tuberose using gibberelic acid and sucrose. Australian Journal of Experimental Agriculture, 41 1227-1230, 2001. 